\title{
Prevalence of Carbapenem Resistance among Gram-Negative Bacteria in a Tertiary Care Hospital in North-East India
}

\author{
Dr. Ph. Henkhoneng Mate, ${ }^{1}$ Dr. Kh. Sulochana Devi, ${ }^{2}$ Dr. Ksh. Mamta Devi, ${ }^{3}$ \\ Dr. San Damrolien, ${ }^{4}$ Dr Ngangom Lilavati Devi, ${ }^{5}$ P. Pratita Devi. \\ ${ }^{1}$ Pgt, ${ }^{2}$ Prof \& HOD, ${ }^{3}$. Asst. Prof, ${ }^{4}$ Demonstrator, ${ }^{5}$ Pgt, ${ }^{6}$. Asst. Prof.) \\ Department of Microbiology, Regional Institute of Medical Sciences, Imphal, Manipur.
}

\begin{abstract}
Summary:
Introduction: Carbapenems are considered to be the last choice for drug resistant Gram-negative bacteria but emergence of carbapenem resistant Gram-negative bacteria worldwide due to the production of carbapenemase has jeopardize their use.

Aims and objectives: To detect the prevalence of carbapenem resistance in Gram-negative bacteria and carbapenemase production by Modified Hodge Test.

Materials and Methods: A total of 160 isolates of Gram-negative bacteria from November 2012 to October 2014, from different clinical samples were included in the study. Those isolates with intermediate or resistant zone to meropenem and doripenem by disc diffusion methods according to CLSI guidelines were confirmed by E-test and were again tested for carbapenemase production by Modified Hodge Test (MHT).

Results: Out of 160 isolates, $48(30 \%)$ showed resistance to carbapenem by disc diffusion and E-test. Carbapenem resistance was higher in female patients $41.6 \%$ than in male patients $15.5 \%$ and among ICU patients $57.1 \%$, ward patients $33.3 \%$ and among OPD patients $13.8 \%$. Maximum number of carbapenem resistant isolate was obtained from urine sample $n=20$, followed by pus $n=13$ and wound swab $n=7$. MHT for carbapenemase production was positive in 29 (60.4\%) of the isolates. Carbapenemase production by MHT was highest with Acinetobacter spp. with $n=5$ (83.3\%), followed by Pseudomonas spp. $n=6$ (75\%) and E.coli spp. $n=16(65.5 \%)$ and nil among Citrobacter spp.
\end{abstract}

Conclusions: Carbapenem resistance due to production of carbapenemase is prevalent in our hospital. MHT is a simple test in the routine lab for detection of carbapenemases.

Key words : Carbapenem, resistance, Gram-negative bacteria, Modified Hodge Test.

\section{Introduction}

Carbapenems are often used as antibiotics of last resort for treating infections due to multidrug-resistant gram-negative bacilli, because they are stable even in response to extended-spectrum and AmpC $\beta$-lactamases. ${ }^{1}$ However, the emergence and proliferation of bacteria resistant to this important group of drug is jeopardizing the use of carbapenems. Resistance to carbapenem mostly is due to production of enzymes-Carbapenemases that hydrolyse carbapenems and other $\beta$-lactams. ${ }^{2}$ Carbapenemase enzymes fall into Ambler classification - A, B and D. ${ }^{3}$ Carbapenemase gene detection by molecular methods is the gold standard but is available in only a few reference laboratories, and phenotypic tests have therefore been developed. ${ }^{4}$ Clinical and Laboratory Standards Institute (CLSI) incorporated the modified Hodge test (MHT) for the detection of carbapenemases. ${ }^{5}$.

\section{Materials And Methods}

The study was done in the Department of Microbiology, Regional Institute of Medical Sciences, Imphal from November 2012 to March 2014 . A total of 160 non-consecutive and non-duplicative isolates of Gram-negative bacteria from different clinical samples were included in the study. The clinical samples included in the study were pus, wound swabs, body fluids, sputum, stool, urine, throat swab, CSF, etc. collected from various patients admitted in wards and those coming to OPDs of the hospital. The bacterial isolates were identified according to standard microbiological procedure. ${ }^{6}$

\section{Antibiotic Susceptibility Testing}

Antbiotic susceptibility testing of Gram-negative isolates was done by Kirby Bauer`s disc diffusion method using the following antibiotics: doripenem, meropenem, ciprofloxacin, amikacin, pipericillin/tazobactam, ceftazidime, nitrofurantoin, norfloxacin, gentamicin, ampicillin, ceftriaxone, amoxyclav, colistin and cotrimoxazole in Mueller Hinton Agar (MHA) according to CLSI guidelines. E.coli strain ATCC 25922 was used as control. ${ }^{7}$ 


\section{Minimum Inhibitory Concentration (MIC)}

MIC of carbapenems was determined by E-test. Those strains which showed reduced susceptibility to doripenem/meropenem in disc diffusion test were confirmed to be carbapenem resistant by E-test (HIMEDIA, INDIA). Resistance of Gram-negative strains to carbapenem was reported if MIC to meropenem and doripenem was $\geq 4 \mu \mathrm{g} / \mathrm{ml}^{8}$

\section{Modified Hodge Test}

The detection of carbapenamase was done by the Modified Hodge Test as suggested by Lee et al ${ }^{13}$ An overnight culture suspension of E. coli ATCC 25922 which was adjusted to one- tenth turbidity of the McFarland 0.5 tube was inoculated evenly on the surface of a Muller-Hinton agar plate using a sterile cotton swab. After a brief drying at room temperature, a meropenem disk $(10 \mu \mathrm{g})$ was placed in the center of the plate. Carbapenem resistant test strains from an overnight culture were streaked heavily from the edge of the disk to the periphery of the plate. The presence of a distorted or clover leaf shaped inhibition zone was interpreted as positive for carbapenemase producing isolates. Klebsiella pneumonia ATCC BAA-1705 was used as control. ${ }^{9}$

\section{Results And Observation}

The study was conducted in the Department of Microbiology, RIMS, Imphal, Manipur from November 2012 to October 2014. A total of 160 Gram-negative bacilli isolated from various clinical samples were taken in the study.

The prevalence of carbapenem resistance among Gram-negative bacterial isolates was 30.0\% (48/160) and sensitivity rate was $70.0 \%$ (111/160). Fig.1. Female patients $55.6 \%$ outnumbered male patients $44.4 \%$. Carbapenem resistance was also higher in female patients $41.6 \%$ than in male patients $15.5 \%$. ( $\mathrm{p}=0.001)$ as shown in table 1.Majority of the patients were from different wards of the hospital (75\%), followed by OPDs (20.6\%) and minimum were from ICUs (4.4\%). But carbapenem resistance rate was highest among ICU patients (57.1\%), followed by ward patients $(33.3 \%)$ and lowest among OPD patients( $13.8 \%)(\mathrm{p}=0.01)$.Table 2.Maximum number of sample was urine $83(51.9 \%)$ followed by pus (23.1\%), sputum (7.5\%), wound swab $(6.2 \%)$ and others (fig 3). Maximum number of carbapenem resistant isolate was obtained from urine sample $\mathrm{n}=$ 20 , followed by pus $\mathrm{n}=13$ and wound swab $\mathrm{n}=7$. Fig 2 .

In this study, it is found that out of 48 carbapenem resistant isolates, 29 (60.4\%) was positive for carbapenemase production by Modified Hodge Test. Carbapenemase production by MHT was highest with Acinetobacter spp. with 5/6 (83.3\%), followed by Pseudomonas spp. 6/8 (75\%) and E.coli spp. 16/26 (65.5\%) and nil among Citrobacter spp. (table 3).

\section{Discussion}

The prevalence of carbapenem resistance among Gram-negative isolates was $30.0 \%$ in this study. Similarly, overall meropenem resistance was about $30 \%$ in a study by Mulla S et al ${ }^{10}$ and $31.81 \%$ by Mahajan $\mathrm{G}$ et al ${ }^{11}$ by disk diffusion method. Some studies recorded lower level of carbapenem resistance. Shivesh $\mathrm{P}$ et al ${ }^{12}$ found $15 \%$ and Shashikala et al ${ }^{13}$ got $10.9 \%$ carbapenem resistance in their respective studies. In a study by Sachinkumar Wankhede et $\mathrm{al}^{14}$ out of 1546 samples screened, $19.40 \%$ of them were carbapenem resistant and Datta $\mathrm{P}$ et al ${ }^{15}$ found that the prevalence of CRE in their Institute is $7.87 \%$. Resistance to carbapenem in this study is low compared to studies by various authors from India. In a study by Bijayini Behera et $\mathrm{al}^{16}$ at the All India Institute of Medical Sciences, New Delhi, carbapenem resistance was found to be 69\%, which much on the higher side.

There was no discordance between two phenotypic methods by Kirby bauer disc diffusion and E-test on carbapenem resistance in this study. In a comparable study by Datta $\mathrm{P}$ et al ${ }^{15}$ twenty-six strains were resistant to carbapenem by both disc-diffusion and E-test. There was no discordance between these two phenotypic methods.

Isolates resistant to meropenem was 30\% (48), while to that of doripenem alone was $26 \%$ (40). This may be due to increased used of meropenem in this area. A study by Mohamudha R.P et al ${ }^{17}$ also reported that among the 45 meropenem resistant isolates, 33 and 21 were resistant to imipenem and ertapenem respectively and Gupta $\mathrm{E}$ et al ${ }^{18}$ also found that overall, resistance to meropenem was 22.16 per cent as compared to imipenem 17.32 per cent $(\mathrm{P}<0.001)$.

In this study maximum sample was from patients of the wards $75.0 \%$ followed by OPDs $20.6 \%$ and ICUs $4.4 \%$ but carbapenem resistance among the isolates is highest in ICUs $57.1 \%$, ward $33.3 .6 \%$ and lowest in OPDs $13.8 \%$. $(\mathrm{p}=0.01)$ Our study is comparable with the study of Dr. Sachinkumar Wankhede et al ${ }^{14}$ where a higher number of MBL producers were from the ICUs (57.63\%) as compared to wards (42.37\%). Similar findings were made by Braykov NP et al ${ }^{19}$ where isolates obtained at ICUs had the highest carbapenem resistant Klebsiella pneumoniae (CRKP) prevalence (aOR, 3.27 [95\% CI, 2.87-3.7] and for $3^{\text {rd }}$ generation cephalosporin and CRKP (G3CRKP), the prevalence of resistance was greatest among isolates obtained at 
nursing homes, followed by isolates obtained at ICUs and then isolates obtained from inpatients and cultures obtained at nursing homes were associated with the highest G3CRKP prevalence, nearly 4 times as high as the prevalence among cultures from outpatient (aOR, 3.9 [95\% CI, 3.71-4.1]).

In another study by Pravin $\mathrm{K}$ et $\mathrm{al}^{20}$ most of the CRE isolates were detected in patient samples from the wards (42\%), then ICU (26\%) and a significant number of isolates was also detected from the OPD patients $(19 \%)$ and Kumar et $\mathrm{al}^{21}$ also found that out of 57 carbapenem resistant cases, 30 (52.6\%) from IPDs, 24 $(42.1 \%)$ from ICUs and $3(5.3 \%)$ from others (triage and OPD).

Maximum number of sample was urine $51.9 \%$ (83/160) followed by pus $23.1 \%$, sputum $7.5 \%$, wound swab 6.2\% and others. In a comparable study Mohamudha R.P et al ${ }^{17}$ also found that the distributions of the sources of the isolates were: urine $37 \%(n=39)$, blood $22.3 \%(n=23)$, wound discharge $11.7(n=12)$, peritoneal fluid 5.8\% ( $\mathrm{n}=6)$, ascitic fluid $10.7 \%(\mathrm{n}=11)$, tracheal aspirate $6.8 \%(\mathrm{n}=7)$, and sputum $4.9 \%(\mathrm{n}=5)$. In the present study, carbapenem resistant organism was isolated mainly from urine $47.1 \%(n=20)$ followed by pus $27.1 \%(n=13)$, wound swabs $14.6 \%(n=7)$, sputum $8.3 \%(n=4)$ and stool $6.3 \%(3)$. Nagaraj $S$ et al ${ }^{22}$ had comparable findings where they observed that the carbapenem-resistant organisms were isolated mainly from urine samples up to $42 \%(n=21)$, followed by wound discharge $18 \%$ and respiratory secretions $16 \%$.

Another study by Yilmaz N O et al ${ }^{23}$ had discordant findings when a total of 38 IMP resistant P. aeruginosa strains were analysed, strains were dominantly isolated from pus $42.1 \%(n=16$,$) , blood cultures$ $26.3 \%(n=10)$, urine $15.8 \%(n=6)$ and tracheal aspirate specimens $15.8 \%(n=6)$. Urine was the most frequent sample received during our study and in most of the studies analysed. The reason for this could be urinary tract infection (UTI), being the most common hospital-acquired infection, which accounts for almost $40 \%$ of all nosocomial infections.

In this study, it is found that out of 48 carbapenem resistant isolates, $60.4 \%$ was positive for carbapenemase production by Modified Hodge Test. Carbapenemase production is highest with Acinetobacter spp. with $83.3 \%$, followed by Pseudomonas spp. $75 \%$ and E.coli spp. $65.5 \%$. None of the 2 Citrobacter spp. was positive for carbapenemase production by MHT or MBL Etest. Carbapenem resistance in these 2 isolates could be due to over production of ESBL or AMPc. In a study by Amjad A et al. ${ }^{24}$ out of 100 isolates which were carbapenem intermediate or sensitive, 69\% showed the presence of carbapenemase by MHT which is similar with this study. In the study by Mahajan $\mathrm{G}$ et al ${ }^{16} 47.6 \%$ of the isolates were found to produce carbapenemase enzyme by MHT and the remaining 22 strains were found to be carbapenemase negative. In a study by Rajkumar MS et al ${ }^{25}$ among the thirty seven meropenem resistant isolates, MHT was positive for $91.89 \%$ of the isolates.

\section{Conclusion}

This report showed that prevalence rate of carbapenem resistance is high in our hospital. Majority of carbapenem resistance here is due to Carbapenemase production. Hence rapid and accurate identification of carbapenem resistance is required for therapeutic and epidemiological reasons so that timely intervention, such as good infection control practices and prudent use of antibiotics will ensure that the spread of carbapenem resistance among organisms is kept under control. The different types of carbapenemases could not be ascertained due to unavailability of PCR in our hospital at the time of this study and perhaps, this is the major limitation in our studies.

\section{Acknowledgement}

I sincerely express my profound gratitude to DBT nodal centre for Medical Colleges and Biomedical Research Institute of Northeast India NAPAAM, Tezpur - 784028, Assam, India for supporting this work under the MD/MS Thesis grant programme.

\section{References}

[1]. Lee K, Lim YS, Yong D, Yum JH, Chong Y. Evaluation of the Hodge Test and the Imipenem-EDT A Double-Disk Synergy Test for Differentiating Metallo- $\beta$-Lactamase-Producing Isolates of Pseudomonas spp. and Acinetobacter spp. J Clin Microbiol 2003;41(10):4623-629.

[2]. Priya D, Varsha G, Shivani G, Jagdish C. Phenotypic method for differentiation of carbapenemases in Enterobacteriaceae: Study from north India.Indian Journal of Pathology and Microbiology. 2012; 55 (3): 357-360.

[3]. Bradford PA, Urban C, Mariano N, Projan SJ, Rahal JJ, Bush K. Imipenem resistance in Klebsiella pneumoniae is associated with the combination of ACT-1, a plasmid mediated AmpC beta-lactamase and the foss of an outer membrane protein. Antimicrob Agents Chemother 1997;41:563-9.

[4]. Andre B, Philippe B, Nathalie G, Catherine D, Dominique D, Guillaume A, Edouard B. Phenotypic Screening of Carbapenemases and Associated $\beta$-Lactamases in Carbapenem-Resistant Enterobacteriaceae. J Clin Microbiol. 2012 Apr; 50(4): 1295-1302.Clinical and Laboratory Standards Institute. Performance Standards for Antimicrobial Susceptibility Testing: Twenty First Informational Supplement M100-S21. CLSI, Wayne, PA: USA; 2011. Available from: https://www.health.gov.au/internet/main/...nsf/.../cdacdi3503c.htm . Accessed on Jan 18, 2014. 
[5]. Weinstein RA, Gupta N, Brandi M. Limbago,Patel JB, Collee JG, et al. Test for the identification of bacteria. In: Collee J G, Fraser AG, Marmion BP, Simmons A, editors. Mackie and McCartney Practical Medical Microbiology. $14^{\text {th }}$ edition. India Elsevier; 2007. p. 151-178.

[6]. Miles RS, Amyes SG. Laboratory control of antimicrobial therapy. In Collee JG, Fraser AG, Marmion BP, Simmons A, editors. Mackie and McCartney Practical Medical Microbiology. $14^{\text {th }}$ edition. India Elsevier; 2007. p. 151-178.

[7]. Koltachchi J, Joan F and Sharon K. Comparison of meropenem MIC by E-test and VITEK-2 in resistant Pseudomas and Acinetobacter isolates. Srilanka journal of Infectious Diseases 2012; 1(2): 28-35.

[8]. Lee K, Chong Y, Shin HB, Kim YA, Yong D, Yum JH. Modified Hodge and disc synergy tests to screen metallo-beta-lactamase producing strains of Pseudomonas spp and Acinetobacter spp. Clin Microbiol Infect 2001; 7:88-91.

[9]. Mulla S, Charan J, Panvala T. Antibiotic sensitivity of Enterobacteriaceae at a tertiary care center in India. Chron Young Sci 2011;2:214-18.

[10]. Mahajan G, Sheemar S, Chopra S, Kaur J, Chowdhary D, Makhija S K. Carbapenem resistance and phenotypic detection of carbapenemases in clinical isolates of Acinetobacter baumannii. Indian J Med Sci 2011;65:18-25.

[11]. Shivesh P. Carbapenem sensitivity profile amongst bacterial isolates from clinical specimens in Kanpur city. Indian J Crit Care Med 2006;10(4):250-53.

[12]. Shashikala, Kanungo R, Srinivasan S, Devi S. Emerging resistance to carbapenems in hospital acquired Pseudomonas infection: A cause for concern. Indian J Pharmacol 2006;38:287-88.

[13]. Sachinkumar W, Vivek I, Ghadge P, Bhore AV. Hospital based infections of Gram- negative organisms. Indian Journal of Basic \& Appl Med Res 2013 June;2(7):797-800.

[14]. Datta P, Gupta V, Garg S, Chander J. Phenotypic method for differentiation of carbapenemases in Enterobacteriaceae: Study from north India. Indian J Pathol Microbiol 2012;55:357-60.

[15]. Bijayini B, Anupam D, Purva M, Arti K. High prevalence of carbapenem resistant Pseudomonas aeruginosa at a tertiary care centre of north India. Are we under-reporting? Indian J Med Res 2008 September; 128:324-25.

[16]. Mohamudha R.P, Harish B.N, Parija S.C. Emerging carbapenem resistance among nosocomial isolates of Klebsiella pneumoniae in South India. Inter Journal Pharma and Bio Sci 2010;6(2).

[17]. Gupta E, Meaty S, Soon S, Dhawan B, Das BK, Kapil A. Emerging resistance to carbapenems in a tertiary care hospital in north India. IJMR 2006 July; 124(1): 95-8.

[18]. Braykov NP, Eber MR, Klein EY, Morgan DJ, Laxminarayan R. Trends in resistance to carbapenems and third-generation cephalosporins among clinicalsolates of Klebsiella pneumoniae in the United States. Infect Control Hosp Epidemiol 2013 Mar;34(3):259-68.

[19]. Pravin K N, Michelle SV. Prevalence of carbapenem resistant Enterobacteriaceae from a tertiary care hospital in Mumbai, Indian Journal of Microbiol and Infect Dis 2013;3(4):207-10.

[20]. Sachin K, Seema B. Leading trend of carbapenem resistance in Enterobacteriaceae in India. Inter Journal of Basic and Appl Med Sci 2014;4(2):205-08.

[21]. Nagaraj S, Chandran SP, Shamanna P, Macaden R. Carbapenem resistance among Escherichia coli and Klebsiella pneumoniae in a tertiary care hospital in South India. IJMM 2012 Jan-Mar;30(1):93-5.

[22]. Yilmaz N O, Agus N, Bozcal E, Uzel A. Prevalence and molecular characterisation of metallo-beta-lactamase producing strains of imipenem-resistant Pseudomonas aeruginosa in Turkey. IJMM 2014;32:349-50.

[23]. Amjad A, Mirza IA, Abbasi SA, Farwa U, Malik N, Zia F. Modified Hodge test: A simple and effective test for detection of carbapenemase production. Iran J Microbiol 2011 December;3(4):189-93.

[24]. Rajkumar MS, Soma S, Puranjay S, Manideepa S. Carbapenemase producing Enterobacteriaceae among urinary isolates: Scenerio from a tertiary care hospital in Eastern India. Journal Evolution Med Dental Sci 2014 February 10;3(6):1323-333.

Figures And Tables

\section{Carbapenem}

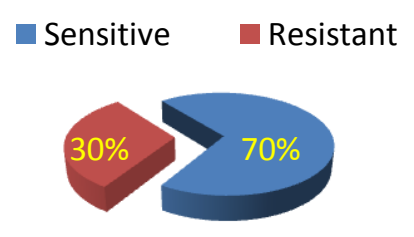

Figure 1: Prevalence of carbapenem resistance in Gram-negative clinical isolates.

Table 1: Distribution of gender.

\begin{tabular}{|l|l|l|l|}
\hline Sex & $\begin{array}{l}\text { Carbapenem resistant } \\
\text { No. Percentage }\end{array}$ & $\begin{array}{l}\text { Carbapenem } \\
\text { sensitive } \\
\text { No. Percentage }\end{array}$ & $\begin{array}{l}\text { Total } \\
\text { No. Percentage }\end{array}$ \\
\hline Male & $11(15.5)$ & $60(84.5)$ & $71(44.0 \%)$ \\
\hline Female & $37(41.6)$ & $52(58.4)$ & $89(55.6)$ \\
\hline Total & $\mathbf{4 8 ( 3 0 )}$ & $\mathbf{1 1 2 ( 7 0 )}$ & $\mathbf{1 6 0}(\mathbf{1 0 0 . 0})$ \\
\hline
\end{tabular}

$\mathrm{X}^{2}=11.315, \mathrm{P}=0.001$ 
Prevalence of carbapenem resistance among Gram-negative bacteria in a tertiary care hospital...

Table 2: Distribution of carbapenem resistant clinical isolates in different locations of the hospital

\begin{tabular}{|c|c|c|c|c|}
\hline \multirow{2}{*}{ Location } & \multicolumn{2}{|c|}{ Carbapenem } & \multirow{3}{*}{$\begin{array}{l}\text { Total } \\
120(75.0)\end{array}$} & \multirow{2}{*}{ CHI SQUARE TEST } \\
\hline & Resistant & nsitive & & \\
\hline Wards & $40(33.3)$ & $80(66.7)$ & & \multirow{4}{*}{$\begin{array}{l}6.33 \\
0.01\end{array}$} \\
\hline OPDs & $4(13.8)$ & $29(86.2)$ & $33(20.6)$ & \\
\hline ICUs & $4(57.1)$ & $3(42.9)$ & $7(4.4)$ & \\
\hline Total & $48(30.0)$ & $112(70.0)$ & $160(100.0)$ & \\
\hline
\end{tabular}

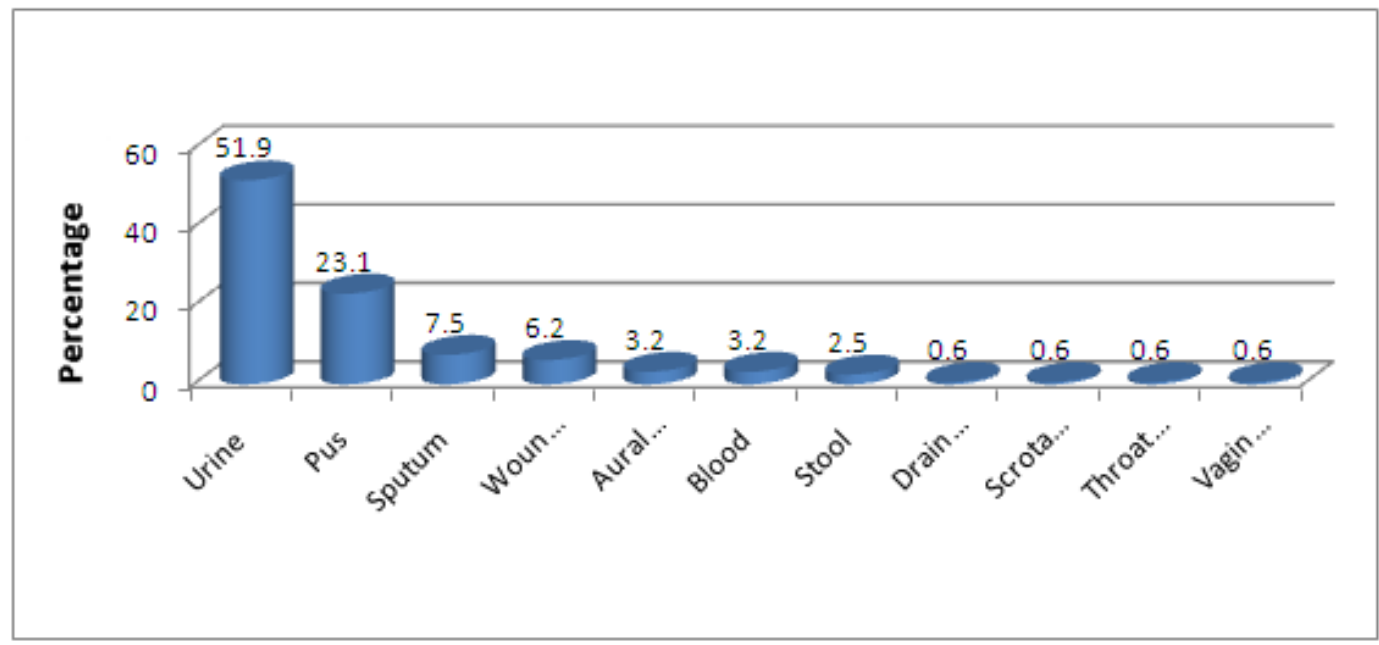

Figure 2: Distribution of the sample

Table 3: Distribution of carbapenemases among the isolates

\begin{tabular}{|l|l|}
\hline $\begin{array}{l}\text { Microorganisms } \\
\text { (No.of carbapenemresistant isolates) }\end{array}$ & $\begin{array}{l}\text { Carbapenemases production by MHT } \\
(\%)\end{array}$ \\
\hline E.coli spp. (26) & $16(61.5)$ \\
\hline Pseudomonas spp. (8) & $6(75)$ \\
\hline Acinetobacter spp. (6) & $5(83.3)$ \\
\hline Klebsiella spp. (3) & $1(33.3)$ \\
\hline Citrobacter spp. (2) & $0(0.0)$ \\
\hline Proteus spp. (2) & $1(50)$ \\
\hline Stenotrophomonas maltophilia (1) & $0(0.0)$ \\
\hline Total & $\mathbf{2 9}(\mathbf{6 0 . 4})$ \\
\hline
\end{tabular}

